\title{
Circulating prolactin and in situ breast cancer risk in the European EPIC cohort: a case-control study
}

Kaja Tikk ${ }^{1,41^{*}}$, Disorn Sookthai ${ }^{1}$, Renée T Fortner ${ }^{1}$, Theron Johnson', Sabina Rinaldi ${ }^{2}$, Isabelle Romieu ${ }^{2}$, Anne Tjønneland ${ }^{3}$, Anja Olsen ${ }^{3}$, Kim Overvad ${ }^{4}$, Françoise Clavel-Chapelon ${ }^{5,6,7}$, Laura Baglietto ${ }^{8,9}$, Heiner Boeing ${ }^{10}$, Antonia Trichopoulou 11,12,13, Pagona Lagiou ${ }^{12,13,14}$, Dimitrios Trichopoulos ${ }^{11,13,14}$, Giovanna Masala ${ }^{15}$, Vittorio Krogh ${ }^{16}$, Rosario Tumino ${ }^{17}$, Fulvio Ricceri ${ }^{18,19}$, Amalia Mattiello ${ }^{20}$, Antonio Agudo ${ }^{21}$, Virginia Menéndez ${ }^{22}$, María-José Sánchez ${ }^{23,27}$, Pilar Amiano ${ }^{24,27}$, Maria-Dolores Chirlaque ${ }^{25,27}$, Aurelio Barricarte ${ }^{26,27}$, HBas Bueno-de-Mesquita 28,29,30,31, Evelyn M Monninkhof ${ }^{32}$, N Charlotte Onland-Moret ${ }^{32}$, Anne Andresson ${ }^{33}$, Malin Sund ${ }^{34}$, Elisabete Weiderpass ${ }^{35,36,37,38}$, Kay-Tee Khaw ${ }^{39}$, Timothy J Key ${ }^{40}$, Ruth C Travis ${ }^{40}$, Melissa A Merritt ${ }^{30}$, Elio Ribolii ${ }^{30}$, Laure Dossus ${ }^{5,6,7}$ and Rudolf Kaaks $^{1}$

\begin{abstract}
Introduction: The relationship between circulating prolactin and invasive breast cancer has been investigated previously, but the association between prolactin levels and in situ breast cancer risk has received less attention.

Methods: We analysed the relationship between pre-diagnostic prolactin levels and the risk of in situ breast cancer overall, and by menopausal status and use of postmenopausal hormone therapy $(\mathrm{HT})$ at blood donation. Conditional logistic regression was used to assess this association in a case-control study nested within the European Prospective Investigation into Cancer and Nutrition (EPIC) cohort, including 307 in situ breast cancer cases and their matched control subjects.
\end{abstract}

Results: We found a significant positive association between higher circulating prolactin levels and risk of in situ breast cancer among all women [pre-and postmenopausal combined, $\mathrm{OR}_{\log 2}=1.35$ (95\% Cl 1.04-1.76), $\mathrm{P}_{\text {trend }}=0.03$ ]. No statistically significant heterogeneity was found between prolactin levels and in situ cancer risk by menopausal status $\left(P_{\text {het }}=0.98\right)$ or baseline HT use $\left(P_{\text {het }}=0.20\right)$, although the observed association was more pronounced among postmenopausal women using HT compared to non-users ( $P_{\text {trend }}=0.06$ vs $P_{\text {trend }}=0.35$ ). In subgroup analyses, the observed positive association was strongest in women diagnosed with in situ breast tumors $<4$ years compared to $\geq 4$ years after blood donation ( $P_{\text {trend }}=0.01$ vs $\left.P_{\text {trend }}=0.63 ; P_{\text {het }}=0.04\right)$ and among nulliparous women compared to parous women $\left(P_{\text {trend }}=0.03\right.$ vs $P_{\text {trend }}=0.15 ; P_{\text {het }}=0.07$ ).

Conclusions: Our data extends prior research linking prolactin and invasive breast cancer to the outcome of in situ breast tumours and shows that higher circulating prolactin is associated with increased risk of in situ breast cancer.

\footnotetext{
* Correspondence: k.tikk@dkfz.de

${ }^{1}$ Division of Cancer Epidemiology, German Cancer Research Center (DKFZ), Heidelberg, Germany

${ }^{41}$ Division of Clinical Epidemiology and Aging Research, German Cancer Research Center (DKFZ) Heidelberg, Im Neuenheimer Feld 581, D-69120 Heidelberg, Germany

Full list of author information is available at the end of the article
} 


\section{Introduction}

In situ breast tumours account for about $20 \%$ of all breast cancers diagnosed by mammography [1] and approximately a third of invasive breast cancers are reported to originate from breast carcinoma in situ [1-3], which may be a precursor of invasive breast cancer [4]. The focus on in situ breast cancer, therefore, offers the advantage of exploring the associations with risk factors that are important early in the carcinogenic process (that is, prior to development of invasive disease) and early in the natural history of breast cancer, increasing our understanding of the aetiology of breast cancer. Identifying common versus distinct risk factors for in situ and invasive disease will, in part, provide insight into the common versus distinct mechanisms through which these cancers develop. Further, in situ breast cancers are relatively aggressively treated with surgery, radiation and/or hormone therapies [1], underscoring the importance of identifying risk factors for this breast cancer subtype.

The relationship between circulating prolactin and invasive breast cancer risk has been investigated previously $[5,6]$. In our previous study we found a modest positive association between circulating prolactin levels and invasive breast cancer risk among postmenopausal women (odds ratio $(\mathrm{OR})_{\mathrm{Q} 4-\mathrm{Q} 1}=1.29(95 \%$ CI 1.05,1.58), $\left.P_{\text {trend }}=0.09\right)[5]$.

The association between prolactin and in situ breast cancer risk, however, has received less attention. In the Nurses' Health Study, the only large-scale prospective study reporting estimates separately for women with invasive and in situ lesions, the increased risk appeared to be confined primarily to invasive cancers $\left(\mathrm{OR}_{\mathrm{Q} 4-\mathrm{Q} 1}=1.38(95 \%\right.$ CI $1.11,1.73), P_{\text {trend }}=0.0005$ for invasive versus $\mathrm{OR}_{\mathrm{Q} 4-\mathrm{Q} 1}=1.16(95 \%$ CI $0.77,1.74)$, $P_{\text {trend }}=0.23$ for in situ breast cancer among postmenopausal women], although there was no heterogeneity comparing in situ versus invasive cancer ( $P$-value for heterogeneity $\left.\left(P_{\text {het }}\right)=0.81\right)[6]$. The only other study to date that included subjects with in situ breast cancer did not provide estimates separately by in situ versus invasive disease [7].

Therefore, in this study we examined the association between pre-diagnostic prolactin concentrations among pre- and postmenopausal women with subsequent risk of in situ breast cancer overall, by menopausal status and by use of postmenopausal hormone therapy (HT) at the time of blood donation within the European Prospective Investigation into Cancer and Nutrition (EPIC) cohort.

\section{Methods}

\section{The EPIC cohort}

The EPIC cohort is based on 366,521 women and 153,457 men recruited between the years 1992 and 2000 in 10 European countries: Denmark, France, Germany, Greece, Italy, Norway, Spain, Sweden, The Netherlands, and the United Kingdom. Details on the subject recruitment, baseline data and blood collection protocols have been reported previously [8]. All EPIC study participants provided written consent for the use of questionnaire information and blood samples in research studies. Ethical approval for the EPIC study was obtained from the ethical review boards of the International Agency for Research on Cancer (IARC) (Lyon, France) and from the local ethics committees in participating countries/study centres (the names of all local ethics committees that approved the study are available Acknowledgements). The current study was additionally approved by the ethical review board at IARC (which holds the central biorepository for EPIC) and the ethical review board in Heidelberg (Heidelberg University), where the laboratory assays and statistical analyses were performed.

Details on vital-status follow up and ascertainment of breast cancer incidence across the study centres were reported previously [9]. Briefly, in all countries (except for France, Greece, and Germany) incident breast cancer cases were identified using a combination of methods employing record linkage with cancer and pathology registries. In Greece, Germany, and France, active follow up of cancer was through health insurance records and direct contact with participants. Self-reported breast cancer cases were all systematically verified from clinical and pathologic records. Breast cancer in situ incidence data were coded according to the International Classification of Disease (ICD-10 code D05). For the present study, the closure date for follow up was the last date of complete follow up for both cancer incidence and vital status, which ranged from 2003 to 2006, depending on the study centre. Sweden was not included in the analysis because independent studies were being completed on breast cancer risk and endogenous hormones [7].

\section{Design of the nested case-control study}

General criteria for the inclusion of cases and matched controls into the study were: (1) women had an available blood sample; (2) could be clearly classified as being either premenopausal or postmenopausal at the time they provided their blood sample, and (3) did not have any previous diagnosis of cancer (except non-melanoma skin cancer). For each case, one matched control (closest to the case, based on matching criteria) with an available blood sample was chosen using incidence density sampling among appropriate risk sets consisting of all cohort members who were alive and free of cancer at the time of diagnosis of the index case. Matching criteria were: study recruitment centre, menopausal status at blood donation, age at blood donation ( \pm 6 months), time at 
blood donation $( \pm 2 \mathrm{~h})$, fasting status, phase of menstrual cycle (matching categories for premenopausal women: early follicular (days 0 to 7 of the cycle), late follicular (days 8 to 11), mid-cycle (days 12 to 16), early luteal (days 17 to 19), mid luteal (days 20 to 24), late luteal (days 25+), and current use of HT at the time of blood donation (for postmenopausal women).

In total, 307 in situ breast cancer cases and an equal number of control subjects were selected for the study. In our analysis, the majority of the cases (91\%) were defined as ductal carcinoma in situ and 9\% as lobular carcinoma in situ or carcinoma in situ NOS (not otherwise specified).

\section{Laboratory assays}

Prolactin assays were performed in the laboratory of the Division of Cancer Epidemiology at the German Cancer Research Centre (DKFZ) and determined by immunoradiometric assay (IRMA (CT), IBL International GMBH, Germany) in blood collected at baseline. The casecontrol pairs were analysed in the same batch and quality controls were included in each analytical batch. Laboratory personnel were blinded to the case-control status of samples and quality controls. The detection range of the assay was 0.35 to $133 \mathrm{ng} / \mathrm{mL}$. The mean inter- and intra-assay coefficients of variation were $4.62 \%$ and $2.17 \%$, respectively.

\section{Statistical analyses}

Conditional logistic regression was used to estimate ORs and $95 \%$ CI for breast cancer in situ occurrence. ORs were calculated across tertiles of circulating prolactin and on a continuous $\log _{2}$ scale. Linear trends for OR estimates were calculated over a continuous $\log _{2}$ scale of prolactin. Circulating prolactin levels vary by menopausal status [10], and therefore the tertile cutpoints were defined separately for pre- and postmenopausal women, based on distributions in the control population.
We decided a priori to adjust all models for parity (nulliparous, parous, or missing data), smoking status (current, never, previous, missing data) and body mass index (BMI) in $\mathrm{kg} / \mathrm{m}^{2}$ (continuous scale), due to modest variation in prolactin levels over these factors in healthy women [10]. A more detailed description of the statistical analysis (including other variables tested for possible confounding) used in this study is described previously [5]. Additionally, for the comparison of the results from our present analysis on in situ breast cancer and our prior results on invasive breast cancer [5], a case-case analysis was performed [11]. All $P$-values presented are two-sided and $P<0.05$ was considered statistically significant. Statistical analyses were conducted using SAS software, version 9.2 (SAS Institute, Cary, NC, USA).

\section{Results}

This nested case-control study consisted of 307 cases diagnosed with in situ breast cancer, and an equal number of controls. For the premenopausal case-control pairs, the median age at blood donation was 46.4 years (range 34.1 to 56.4 years), and the median age at diagnosis for the cases was 50.6 years (range 37.3 to 61.3) (Table 1). Among postmenopausal women the ages at blood donation and diagnosis were 58.1 years (range 47.7 to 71.4 ) and 62.8 years (range 51.0 to 78.3 ), respectively. The median follow-up time between blood donation and date of diagnosis was 4.2 years (range 0.02 to 10.7 years). This study included 82 postmenopausal case-control pairs who used HT at the time they provided their baseline blood sample. Out of 307 in situ cases selected to this analysis, 15 patients developed invasive breast cancer after diagnosis of in situ disease during further follow up. The median levels of prolactin were $8.3 \mathrm{ng} / \mathrm{mL}$ (range 3.8 to $28.1 \mathrm{ng} / \mathrm{mL}$ ) for patients who developed invasive disease after diagnosis of in situ disease and

Table 1 Baseline characteristics of in situ breast cancer case and control subjects (data presented as median [min, max] or $n[\%])$

\begin{tabular}{|c|c|c|c|c|}
\hline & \multicolumn{2}{|c|}{ Premenopausal women* } & \multicolumn{2}{|c|}{ Postmenopausal women* } \\
\hline & Cases $(n=86)$ & Controls $(n=86)$ & Cases $(n=221)$ & Controls $(n=221)$ \\
\hline Age at blood donation, years & $46.4(34.1,56.4)$ & $46.5(34.4,55.6)$ & $58.1(47.7,70.6)$ & $58.0(47.7,71.4)$ \\
\hline Age at menopause, years & & & $50.0(33.0,59.0)$ & $50.0(29.0,58.0)$ \\
\hline Age at diagnosis, years & $50.6(37.3,61.3)$ & & $62.8(51.0,78.3)$ & \\
\hline Lag time till diagnosis, years & $4.1(0.02,10.0)$ & & $4.4(0.02,10.7)$ & \\
\hline Body mass index, $\mathrm{kg} / \mathrm{m}^{2}$ & $23.3(17.1,32.8)$ & $24.0(17.4,37.2)$ & $24.9(17.8,40.8)$ & $24.5(18.2,45.9)$ \\
\hline Ever had a full-term pregnancy & $68(82.9)$ & $70(81.4)$ & $190(86.8)$ & $188(85.5)$ \\
\hline Baseline smoking & $15(17.4)$ & $20(23.3)$ & $25(11.3)$ & $33(14.9)$ \\
\hline Baseline use of hormone therapy & & & $82(37.1)$ & $82(37.1)$ \\
\hline Prolactin levels, ng/mL & $8.9(3.4,51.7)$ & $7.7(2.9,133.0)$ & $6.2(2.6,128.3)$ & $5.9(2.6,55.3)$ \\
\hline
\end{tabular}

Data are presented as median (minimum, maximum) or number (\%). *Menopausal status at the time of blood donation. 
$6.8 \mathrm{ng} / \mathrm{mL}$ (range 2.6 to $128.3 \mathrm{ng} / \mathrm{mL}$ ) in patients who did not develop invasive disease during further follow up (data not shown).

In conditional logistic regression analyses adjusting for parity, smoking status, and BMI, there was a positive association between prolactin concentrations and in situ breast cancer risk among all women (pre-and postmenopausal combined), with a statistically significant $35 \%$ increase in risk for each one unit increase in prolactin on the continuous $\log _{2}$ scale $\left(\mathrm{OR}_{\log 2}=1.35\right.$ (95\% CI 1.04, 1.76), $\left.P_{\text {trend }}=0.03\right)$ (Table 2). The association was similar comparing the top to the bottom tertiles of prolactin, but did not reach statistical significance $\left(\mathrm{OR}_{\mathrm{Q} 3-\mathrm{Q} 1}=1.37\right.$ $(95 \%$ CI $0.87,2.16))$. We observed no statistically significant heterogeneity in stratified analyses by menopausal status $\left(P_{\text {het }}=0.98\right)$. However, after additional stratification by baseline HT use among postmenopausal women, we observed a suggestive association among the HT users $\left(\mathrm{OR}_{\log 2}=1.77\right.$ (95\% CI 0.98, 3.21), $\left.P_{\text {trend }}=0.06\right)$, but not among the non-users $\left(\mathrm{OR}_{\log 2}=1.20(95 \% \mathrm{CI}\right.$ $0.82,1.76), \mathrm{P}_{\text {trend }}=0.35$ ). However, case numbers in these subgroups were small, CIs overlapping and the test for heterogeneity between HT users and non-users was not statistically significant $\left(P_{\text {het }}=0.20\right)$.

In subgroup analyses (Table 3 ), we observed significant heterogeneity in the strength of the association with in situ breast cancer risk by time between blood donation and diagnosis (that is, lag time) among all women $\left(P_{\text {het }}=0.04\right)$. Higher concentrations of prolactin were significantly associated with in situ breast cancer diagnosed less than 4 years since blood donation $\left(\mathrm{OR}_{\log 2}=1.78\right.$ (95\% CI 1.12, 2.84), $\left.P_{\text {trend }}=0.01\right)$, but not with breast cancer diagnosed 4 or more years since blood donation $\left(\mathrm{OR}_{\log 2}=1.09\right.$ (95\% CI 0.77, 1.55), $P_{\text {trend }}=0.63$; $\left.P_{\text {het }}=0.04\right)$. Similarly, the estimated association of prolactin with in situ breast cancer risk appeared stronger among nulliparous women compared to parous women, more pronounced in analysis restricted to postmenopausal women $\left(\mathrm{OR}_{\log 2}=5.10(95 \%\right.$ CI $1.23,21.15)$, $P_{\text {trend }}=0.02$ in nulliparous women versus $\mathrm{OR}_{\log 2}=1.22$ (95\% CI $0.88,1.68), P_{\text {trend }}=0.24$ in parous women; $P_{\text {het }}=0.02$ ). It should be noted, however, that the case numbers for nulliparous women were small and the CIs for risk estimates wide. There was no significant heterogeneity by age at tumour diagnosis in either premenopausal or postmenopausal women $\left(P_{\text {het }}=0.87\right)$ (data not shown).

Additional statistical analyses showed no evidence for major confounding effects by other lifestyle and reproductive factors nor was there an interaction between prolactin and BMI $\left(P_{\text {interaction }}=0.84\right)$ (data not shown).

Finally, we compared the results from our present analysis on in situ breast cancer and from our prior analysis on invasive breast cancer [5]. The median levels of prolactin comparing in situ $(\mathrm{n}=307)$ versus invasive

Table 2 Adjusted odd ratios ${ }^{a}$ (OR) for in situ breast cancer by tertile levels and on a continuous log scale of $_{2}$ circulating prolactin

\begin{tabular}{|c|c|c|c|c|c|c|}
\hline & & Tertiles & & $\log _{2}$ & & \\
\hline & 1 & 2 & 3 & Odds ratio $(95 \% \mathrm{Cl})$ & $P_{\text {trend }}^{\mathrm{b}}$ & $P_{\text {het }}^{c}$ \\
\hline All won & & & & & & \\
\hline $\mathrm{Ca} / \mathrm{Co}$ & $89 / 101$ & $94 / 104$ & $124 / 102$ & & & \\
\hline OR & Ref. & $0.98(0.65,1.49)$ & $1.37(0.87,2.16)$ & $1.35(1.04,1.76)$ & 0.03 & \\
\hline Premen & & & & & & \\
\hline $\mathrm{Ca} / \mathrm{Co}$ & $27 / 29$ & $19 / 28$ & $40 / 29$ & & & \\
\hline OR & Ref. & $0.63(0.25,1.58)$ & $1.49(0.59,3.74)$ & $1.30(0.80,2.10)$ & 0.28 & \\
\hline Postme & & & & & & \\
\hline $\mathrm{Ca} / \mathrm{Co}$ & $65 / 72$ & $69 / 75$ & $87 / 74$ & & & 0.98 \\
\hline OR & Ref. & $1.00(0.61,1.63)$ & $1.31(0.77,2.22)$ & $1.38(1.00,1.91)$ & 0.05 & \\
\hline Postme & & & & & & \\
\hline $\mathrm{Ca} / \mathrm{Co}$ & $50 / 51$ & $39 / 49$ & $50 / 39$ & & & \\
\hline OR & Ref. & $0.84(0.44,1.6)$ & $1.27(0.65,2.47)$ & $1.20(0.82,1.76)$ & 0.35 & \\
\hline Postme & & & & & & \\
\hline $\mathrm{Ca} / \mathrm{Co}$ & $15 / 21$ & $30 / 26$ & $37 / 35$ & & & 0.20 \\
\hline OR & Ref. & $1.63(0.70,3.81)$ & $1.62(0.64,4.09)$ & $1.77(0.98,3.21)$ & 0.06 & \\
\hline
\end{tabular}

${ }^{a}$ Adjusted for parity (nulliparous, parous, or missing data), smoking status (current, never, previous, or missing data) and body mass index (continuous scale); ${ }^{b}$ linear trends for odds ratio estimates over a continuous scale of prolactin levels; ${ }^{c}$ statistical tests for heterogeneity were based on the likelihood-ratio test, comparing the model fit for logistic regression models with and without a corresponding interaction term. Ca/Co, Case/Control; Ref., reference; HT, hormone therapy. 
Table 3 Adjusted odds ratios ${ }^{\mathrm{a}}$ (OR) for in situ breast cancer on a continuous $\log _{2}$ scale of circulating prolactin by subgroup analysis

$\begin{array}{lll}\text { Case-control } \log _{2} \text { odds } & P_{\text {trend }}^{\mathrm{b}} P_{\text {het }}^{\mathrm{c}}\end{array}$

All women

Lag time till diagnosis

$\begin{array}{lllll}<4 \text { years } & 127 & 1.78(1.12,2.84) & 0.01 & 0.04 \\ \geq 4 \text { years } & 180 & 1.09(0.77,1.55) & 0.63 & \\ \begin{array}{l}\text { Parity } \\ \text { d }\end{array} & & & & \\ \text { Nulliparous } & 42 / 45 & 2.64(1.07,6.51) & 0.03 & 0.07 \\ \text { Parous } & 251 / 251 & 1.21(0.94,1.57) & 0.15 & \end{array}$

Premenopausal women

Lag time till diagnosis

$\begin{array}{lllll}<4 \text { years } & 39 & 2.30(0.85,6.14) & 0.10 & 0.15 \\ \geq 4 \text { years } & 47 & 0.97(0.51,1.83) & 0.92 & \end{array}$

Postmenopausal women (all)

Lag time till diagnosis

$\begin{array}{lllll}<4 \text { years } & 88 & 1.66(0.97,2.85) & 0.07 & 0.19 \\ \geq 4 \text { years } & 133 & 1.18(0.76,1.82) & 0.46 & \\ \begin{array}{l}\text { Parity }{ }^{d} \\ \text { Nulliparous }\end{array} & 29 / 32 & \begin{array}{l}5.10(1.23, \\ 21.15)\end{array} & 0.02 & 0.02 \\ & & 1.22(0.88,1.68) & 0.24 & \end{array}$

Postmenopausal non-HT users

Lag time till diagnosis

$\begin{array}{lllll}<4 \text { years } & 52 & 1.20(0.70,2.05) & 0.51 & 0.52 \\ \geq 4 \text { years } & 87 & 1.08(0.61,1.93) & 0.79 & \end{array}$

Postmenopausal

HT users

Lag time till diagnosis

\begin{tabular}{lllll}
$<4$ years & 36 & $6.02(1.31$, & 0.02 & 0.04 \\
& & $27.72)$ & & \\
$\geq 4$ years & 46 & $1.29(0.64,2.61)$ & 0.47 & \\
\hline
\end{tabular}

${ }^{a}$ Adjusted for parity (nulliparous, parous, missing data), smoking status (current, never, previous, or missing data) and body mass index (continuous scale); 'blinear trends for odds ratio estimates over a continuous scale of prolactin levels; ' heterogeneity between subgroups was based on the chi-square test; ${ }^{\mathrm{d}}$ using unconditional logistic regression, adjusted for age and time at blood donation, fasting and smoking status, body mass index, phase of menstrual cycle for premenopausal women and current use of hormone therapy (HT) for postmenopausal women.

breast cancer tumors $(\mathrm{n}=2250)$ were 8.9 (in situ) versus $8.6 \mathrm{ng} / \mathrm{mL}$ (invasive) for premenopausal women and 6.2 (in situ) versus 6.1 (invasive) $\mathrm{ng} / \mathrm{mL}$ for the postmenopausal women (data not shown). A heterogeneity test comparing in situ versus invasive disease was not statistically significant $\left(P_{\text {het }}=0.25\right.$ for premenopausal women and $P_{\text {het }}=0.33$ for postmenopausal women).

\section{Discussion}

In this prospective study, we observed a significant positive association between pre-diagnostic circulating prolactin levels and risk of in situ breast cancer. Our data showed no evidence for heterogeneity in the relationship of prolactin levels and in situ cancer risk by menopausal status or postmenopausal HT use at blood donation, although the observed positive association was more pronounced among $\mathrm{HT}$ users versus non-users. In addition, the associations were strongest among women diagnosed with in situ breast tumors less than 4 years after blood donation and also among nulliparous women.

Our findings of modest, positive associations between prolactin and in situ breast cancer risk are similar to those observed for invasive breast cancer. Prior analyses limited to invasive cases found modest significant associations between circulating prolactin and risk of invasive breast cancer, with up to a $50 \%$ increase in risk contrasting top versus bottom quartiles [5,6]. Moreover, comparable to our previous findings for invasive breast cancer [5], the observed association in the present study of in situ breast cancer was more pronounced among postmenopausal HT users as compared to non-users. Although in situ carcinomas in the breast may not have invasive characteristics [12], up to 50\% of in situ lesions, if left untreated, progress to invasive disease [3]. Little is known about the exact mechanisms influencing the possible progression of in situ lesions to invasive disease. However, the very similar associations between prolactin and breast cancer risk observed in our previous study of invasive disease [5] and the current study of in situ disease suggest that circulating prolactin may influence risk of invasive and in situ breast cancer via the same aetiological pathway. Prolactin may also play a role in progression from in situ to invasive breast cancer. However, out of 307 in situ cases selected for this analysis, only 15 developed invasive breast cancer during further follow up. Therefore, we were unable to assess the association between prolactin and progression from in situ to invasive disease in this study.

Although a vast body of evidence from animal, in vitro, and epidemiological studies strongly supports the involvement of prolactin in breast cancer development $[5,6,13,14]$, the complex and diverse biological and molecular mechanisms through which prolactin may increase risk of breast cancer are not clear. Some proposed mechanisms include its proliferative effects on malignant breast cells, mitogenic action, and inhibition of apoptosis via signalling through the prolactin receptor [14]. In addition to the endocrine (circulating) concentrations, locally produced prolactin may promote cancer development via autocrine and paracrine effects [15]. Evidence is also emerging that drugs resulting in 
elevated prolactin (for example, neuroleptic and hormonal medications) may increase breast cancer risk [16,17].

The relationship between prolactin and in situ breast cancer risk was confined to tumors diagnosed within the first 4 years from blood donation. Interestingly, this differential association by time between blood collection and diagnosis was not evident for invasive breast cancer risk in our previous analysis [5]. Whether prolactin is involved in the early development or promotes late-stage growth of an established tumor is unclear. Consistent with the current analysis, recent results from the Nurses' Health Study suggest that prolactin primarily plays a role in the late stage of tumor development, with stronger positive associations observed among participants providing blood samples closer to diagnosis $[6,18]$. This is also supported by studies observing elevated levels of circulating prolactin in breast cancer patients [19], and demonstrating the ability of breast tumors to secrete prolactin [13]. However, our present analysis suggests that prolactin may also operate early in the natural history of breast cancer by increasing risk of in situ tumors, the earliest detectable breast carcinomas.

In the current study, prolactin was associated with in situ breast cancer among postmenopausal nulliparous women but not in parous women. Parity, a wellestablished protective factor for breast cancer [20], has been associated with a long-term post-pregnancy reduction in levels of circulating prolactin [10,21]. Our results support the hypothesis that lowered prolactin levels following pregnancy might serve as one of several possible mechanisms that mediate the long-term reduction in risk afforded by parity. However, our findings should be interpreted with caution as only about $15 \%$ of women in this case-control study were nulliparous and OR estimates for nulliparous women had wide confidence limits.

Our study is the second largest prospective study on the association between prolactin and breast cancer risk, to provide risk estimates for in situ breast cancer. A general limitation of our study is that prolactin levels were measured only at a single point in time. However, reliability studies in which repeat blood samples were taken over intervals of up to three years have shown relatively high intra-class correlation (ICC coefficients of up to 0.76 ) between individuals' serum prolactin levels over time [22-24]. In situ breast cancer is largely diagnosed as a result of mammography; however, we were not able to adjust our models for the participation in mammographic screening, nor were we able to compare rates of screening in cases and controls. Our study had relatively limited numbers of case subjects for subgroup analysis, and thus the statistical power for detecting associations of prolactin with risk in these subgroups was limited. Due to very limited information available for the molecular characteristics of in situ tumors, we were not able to perform the analysis by molecular subtypes of the lesions.

\section{Conclusions}

In conclusion, we have extended prior research showing an association between prolactin and invasive breast cancer to the outcome of in situ breast cancer. Our study shows that higher circulating levels of prolactin may be associated with increased risk of in situ breast cancer. It is estimated that up to $50 \%$ of untreated in situ breast lesions will progress to invasive disease. However, we are currently not able to distinguish between indolent and more aggressive in situ carcinomas, and the side effects of the medical management of in situ lesions (for example, lumpectomy, radiation and/or hormone therapy) are not negligible. Therefore, identification of risk factors for in situ breast carcinomas is important both from the perspective of characterizing the risk of the disease as diagnosed and treated, as well as providing insight into the aetiology of the in situ carcinomas, which represent pre-invasive disease. Additional work to characterize circulating prolactin and risk of invasive disease, particularly after the development of in situ breast disease, is needed.

\section{Abbreviations \\ BMI: body mass index; EPIC: European Prospective Investigation into Cancer and Nutrition; HT: hormone therapy; IARC: International Agency for Research on Cancer; ICC: intra-class correlation; ICD: International Classification of Diseases for Oncology; OR: odds ratio; Q4-Q1: Quartile4-Quartile1.}

\section{Competing interests}

The authors declare that they have no competing interests.

\section{Authors' contributions}

$K T$, DS and RK contributed to the conception of the current analysis and $S R$, IR, AT, KO, FC-C, HB, AT, DT, VK, RT, M-JS, M-DC, AB, HBB, EM, CO-M, AA, EW, $K-T K, T K, R T, M M, E R, L D$, and RK were involved in the design and acquisition of data from the EPIC cohort. TJ carried out the immunoassays. KT, DS and RK contributed to the analysis and $K T, D S, R F, S R, I R, A T, A O, K O, F C-C, L B, H B$, AT, PL, DT, GM, VK, RT, FR, AM, AA, VM, PA, M-JS, M-DC, AB, HBB, EM, CO-M, $A A, M S, E W, K-T K, T K, R T, M M, E R, L D$, and RK contributed to the interpretation of the data. KT, RF and RK drafted the manuscript and DS, TJ, SR, IR, AT, AO, KO, FC-C, LB, HB, AT, PL, DT, GM, VK, RT, FR, AM, AA, VM, PA, M-JS, M-DC, AB, HBB, EM, CO-M, AA, MS, EW, K-TK, TK, RT, MM, ER, and LD revised the final draft critically for important critical content. All authors read and approved the final manuscript.

\section{Acknowledgements}

We thank all the EPIC cohort participants. Furthermore, we thank Britta Lederer and Sigrid Henke for their work in conducting the immunoassays and Bertrand Hémon for his help with the EPIC database. Ethical approval for the EPIC study was obtained from the ethical review boards of IARC (Lyon, France) and from The Committee of Bioethics and Deontology of the Hellenic Health Foundation (Athens; Greece), Norwich District Ethics Committee (Cambridge; UK), The National Committee on Health Research (Aarhus, Copenhagen; Denmark), Ethics Comité de Protection des Personnes (Paris; France), Ethics Committee of the Heidelberg University Medical School (Heidelberg; Germany), IARC Ethics Committee (France), Imperial College Research Ethics Committee (ICREC) (London; UK), Comitato Etico Indipendente, Fondazione IRCCS Istituto Nazionale dei Tumori, Milano (Milan, Naples, Ragusa, Turin; Italy), Comitato Etico Locale Azienda Sanitaria di Firenze (Florence; Italy), 
Ethics Committee of Lundst University (Malmo; Sweden), The Medical Ethical Committee (METC, Medisch Ethische Toetsingscommissie) of the University Medical Center Utrecht (UMCU)( Utrecht, Bilthoven; the Netherlands), The Regional Committee for Medical and Health Research Ethics, North Norway (REK nord) (Norway), Scotland A Research Ethics Committee (Oxford; UK), Ethikkommission der Landesärztekammer Brandenburg Cottbus, (Potsdam; Germany), CEIC Comité de Ética de Investigación Clínica (Asturias, Barcelona, Granada, Murcia, Navarre, San Sebastian; Spain), Human Genetics Foundation Torino: Ethics Committee (Torino; Italy), Umea Regional Ethical Review Board (Umea, Sweden). The coordination of EPIC is financially supported by the European Commission (DG-SANCO) and the International Agency for Research on Cancer. The national cohorts are supported by Danish Cancer Society (Denmark); Ligue contre le Cancer, Mutuelle Générale de l'Éducation Nationale, Institut National de la Santé et de la Recherche Médicale (France); Deutsche Krebshilfe, Deutsches Krebsforschungszentrum and Federal Ministry of Education and Research (Germany); the Hellenic Health Foundation (Greece); Italian Association for Research on Cancer (AIRC) and National Research Council (Italy); Dutch Ministry of Public Health, Welfare and Sports (WWS), Netherlands Cancer Registry (NKR), LK Research Funds, Dutch Prevention Funds, Dutch ZON (Zorg Onderzoek Nederland), World Cancer Research Fund (WCRF), Statistics Netherlands (The Netherlands); Norwegian Research Council, Norwegian. Cancer Society, ERC-2009-AdG 232997 and Nordforsk, Nordic Centre of Excellence programme on Food, Nutrition and Health. (Norway); Health Research Fund (FIS), The Spanish Ministry of Health (ISCIII RETICC RD06/ 0020/0091) and the Catalan Institute of Oncology, Regional Governments of Andalucía, Asturias, Basque Country, Murcia (no 6236) and Navarra, ISCIII RETIC (RD06/0020; Spain); Swedish Cancer Society, Swedish Scientific Council and Regional Government of Skåne and Västerbotten (Sweden); Cancer Research UK, and the Medical Research Council (UK).

\section{Funding}

This research did not receive any specific grant from any funding agency in the public, commercial or not-for-profit sector.

\section{Author details}

'Division of Cancer Epidemiology, German Cancer Research Center (DKFZ), Heidelberg, Germany. ${ }^{2}$ Section of Nutrition and Metabolism, International Agency for Research on Cancer (IARC), Lyon, France. ${ }^{3}$ Danish Cancer Society Research Center, Copenhagen, Denmark. ${ }^{4}$ Section for Epidemiology, Department of Public Health, Aarhus University, Aarhus, Denmark. ${ }^{5}$ INSERM, Centre for Research in Epidemiology and Population Health [CESP], U1018, Nutrition, Hormones and Women's Health team, F-94805 Villejuif, France. ${ }^{6}$ University Paris Sud, UMRS 1018, F-94805 Villejuif, France. ${ }^{7}$ IGR, F-94805 Villejuif, France. ${ }^{8}$ Cancer Epidemiology Centre, Cancer Council Victoria, 3053 Melbourne, Australia. ${ }^{9}$ Centre for Molecular, Environmental, Genetic, and Analytic Epidemiology, The University of Melbourne, 3010 Melbourne, Australia. ${ }^{10}$ Department of Epidemiology, German Institute of Human Nutrition (DIfE) Potsdam-Rehbrücke, Nuthetal, Germany. ${ }^{11}$ Hellenic Health Foundation, 13 Kaisareias Street, GR-115 27 Athens, Greece. ${ }^{12}$ Department of Hygiene, Epidemiology and Medical Statistics, University of Athens Medical School, 75 M. Asias StreetGoudi GR-115 27 Athens, Greece. ${ }^{13}$ Bureau of Epidemiologic Research, Academy of Athens, 23 Alexandroupoleos Street, Athens GR-115 27, Greece. ${ }^{14}$ Department of Epidemiology, Harvard School of Public Health, 677 Huntington Avenue, Boston, USA. ${ }^{15}$ Molecular and Nutritional Epidemiology Unit, Cancer Research and Prevention Institute ISPO, Florence, Italy. ${ }^{16}$ Epidemiology and Prevention Unit, Fondazione IRCCS Istituto Nazionale dei Tumori, Via Venezian 1, Milano, Italy. ${ }^{17}$ Cancer Registry and Histopathology Unit, "Civic - M.P.Arezzo" Hospital ASP, Ragusa, Italy. ${ }^{18}$ Unit of Cancer Epidemiology, AO Citta' della Salute e della Scienza, University of Turin, Torino, Italy. ${ }^{19}$ Center for Cancer Prevention (CPO), Via Santena 7, 10126 Torino, Italy. ${ }^{20}$ Department of Clinical and Experimental Medicine, Federico II University, Naples, Italy. ${ }^{21}$ Unit of Nutrition, Environment and Cancer, Catalan Institute of Oncology-ICO, IDIBELL, L'Hospitalet de Llobregat, Barcelona 08908, Spain. ${ }^{22}$ Public Health Directorate, Asturias, Spain. ${ }^{23}$ Escuela Andaluza de Salud Pública, Instituto de Investigación Biosanitario de Granada (Granada.ibs), Hospitales Universitarios de Granada/Universidad de Granada, Granada, Spain. ${ }^{24}$ Public Health Division of Gipuzkoa, BioDonostia Reserach Institute, San Sebastian, Spain. ${ }^{25}$ Department of Epidemiology, Murcia Regional Health Authority, Murcia, Spain. ${ }^{26}$ Navarre Public Health Institute, Pamplona, Spain. ${ }^{27}$ Consortium for Biomedical Research in Epidemiology and Public Health (CIBER de Epidemiología y Salud
Pública (CIBERESP)), Madrid, Spain. ${ }^{28}$ Department for Determinants of Chronic Diseases (DCD), National Institute for Public Health and the Environment (RIVM), Bilthoven, The Netherlands. ${ }^{29}$ Department of Gastroenterology and Hepatology, University Medical Centre, Utrecht, The Netherlands.

${ }^{30}$ Department of Epidemiology and Biostatistics, The School of Public Health, Imperial College, London, UK. ${ }^{31}$ Department of Social \& Preventive Medicine, Faculty of Medicine, University of Malaya, Kuala Lumpur, Malaysia. ${ }^{32}$ Department of Epidemiology, Julius Center for Health Sciences and Primary Care, University Medical Center Utrecht, Utrecht, The Netherlands. ${ }^{33}$ Department of Radiation Sciences, University of Umeå, Umeå, Sweden. ${ }^{34}$ Department of Surgical and Perioperative Sciences, Umeå University, Umeå, Sweden. ${ }^{35}$ Department of Community Medicine, Faculty of Health Sciences, University of Troms $\varnothing$, Troms $\varnothing$, Norway. ${ }^{36}$ Department of Research, Cancer Registry of Norway, Oslo, Norway. ${ }^{37}$ Department of Medical Epidemiology and Biostatistics, Karolinska Institutet, Stockholm, Sweden. ${ }^{38}$ Samfundet Folkhälsan, Helsinki, Finland. ${ }^{39}$ School of Clinical Medicine, University of Cambridge, Cambridge, UK. ${ }^{40}$ Cancer Epidemiology Unit, Nuffield Department of Population Health, University of Oxford, Oxford, UK. ${ }^{41}$ Division of Clinical Epidemiology and Aging Research, German Cancer Research Center (DKFZ) Heidelberg, Im Neuenheimer Feld 581, D-69120 Heidelberg, Germany.

\section{Received: 31 July 2014 Accepted: 17 March 2015}

\section{Published online: 31 March 2015}

\section{References}

1. Leonard GD, Swain SM. Ductal carcinoma in situ, complexities and challenges. J Natl Cancer Inst. 2004;96:906-20.

2. Sanders ME, Schuyler PA, Dupont WD, Page DL. The natural history of low-grade ductal carcinoma in situ of the breast in women treated by biopsy only revealed over 30 years of long-term follow-up. Cancer. 2005;103:2481-4.

3. Jones JL. Overdiagnosis and overtreatment of breast cancer: progression of ductal carcinoma in situ: the pathological perspective. Breast Cancer Res. 2006:8:204.

4. Reeves GK, Pirie K, Green J, Bull D, Beral V, For the Million Women Study C. Comparison of the effects of genetic and environmental risk factors on in situ and invasive ductal breast cancer. Int J Cancer. 2012;131:930-7.

5. Tikk K, Sookthai D, Johnson T, Rinaldi S, Romieu I, Tjønneland A, et al. Circulating prolactin and breast cancer risk among pre- and postmenopausal women in the EPIC cohort. Ann Oncol. 2014;25:1422-8.

6. Tworoger SS, Eliassen AH, Zhang X, Qian J, Sluss PM, Rosner BA, et al. A 20-year prospective study of plasma prolactin as a risk marker of breast cancer development. Cancer Res. 2013;73:4810-9.

7. Manjer J, Johansson R, Berglund G, Janzon L, Kaaks R, Agren A, et al. Postmenopausal breast cancer risk in relation to sex steroid hormones, prolactin and SHBG (Sweden). Cancer Causes Control. 2003;14:599-607.

8. Riboli E, Hunt KJ, Slimani N, Ferrari P, Norat T, Fahey M, et al. European Prospective Investigation into Cancer and Nutrition (EPIC): study populations and data collection. Public Health Nutr. 2002;5:1113-24.

9. James RE, Lukanova A, Dossus L, Becker S, Rinaldi S, Tjonneland A, et al. Postmenopausal serum sex steroids and risk of hormone receptor-positive and -negative breast cancer: a nested case-control study. Cancer Prev Res. 2011;4:1626-35.

10. Tikk K, Sookthai D, Johnson T, Dossus L, Clavel-Chapelon F, Tjønneland A, et al. Prolactin determinants in healthy women: a large cross-sectional study within the EPIC cohort. Cancer Epidemiol Biomarkers Prev. 2014;23:2532-42.

11. Wang M, Spiegelman D, Kuchiba A, Lochhead P, Kim S, Chan AT, et al. Conceptual and Statistical Frameworks in Epidemiology for Studying Disease Heterogeneity and Pathogenesis. http://cdn1.sph.harvard.edu/wpcontent/uploads/sites/271/2014/04/subtype_2014.pdf.

12. Muggerud AA, Hallett M, Johnsen H, Kleivi K, Zhou W, Tahmasebpoor S, et al. Molecular diversity in ductal carcinoma in situ (DCIS) and early invasive breast cancer. Mol Oncol. 2010;4:357-68.

13. Ginsburg E, Vonderhaar BK. Prolactin synthesis and secretion by human breast cancer cells. Cancer Res. 1995;55:2591-5.

14. Clevenger CV, Furth PA, Hankinson SE, Schuler LA. The role of prolactin in mammary carcinoma. Endocr Rev. 2003;24:1-27.

15. Bernichtein S, Touraine P, Goffin V. New concepts in prolactin biology. J Endocrinol. 2010;206:1-11. 
16. Wang PS, Walker AM, Tsuang MT, Orav EJ, Glynn RJ, Levin R, et al. Dopamine antagonists and the development of breast cancer. Arch Gen Psychiatry. 2002;59:1147-54.

17. Chen WY. Postmenopausal hormone therapy and breast cancer risk: current status and unanswered questions. Endocrinol Metab Clin North Am. 2011:40:509-18.

18. Tworoger SS, Sluss P, Hankinson SE. Association between plasma prolactin concentrations and risk of breast cancer among predominately premenopausal women. Cancer Res. 2006;66:2476-82.

19. Faupel-Badger JM, Sherman ME, Garcia-Closas M, Gaudet MM, Falk RT, Andaya A, et al. Prolactin serum levels and breast cancer: relationships with risk factors and tumour characteristics among pre- and postmenopausal women in a population-based case-control study from Poland. Br I Cancer. 2010;103:1097-102.

20. Albrektsen G, Heuch I, Hansen S, Kvale G. Breast cancer risk by age at birth time since birth and time intervals between births: exploring interaction effects. Br J Cancer. 2004;92:167-75.

21. Eliassen $A H$, Tworoger SS, Hankinson SE. Reproductive factors and family history of breast cancer in relation to plasma prolactin levels in premenopausal and postmenopausal women. Int J Cancer. 2007;120:1536-41.

22. Hankinson SE, Manson JE, Spiegelman D, Willett WC, Longcope C, Speizer FE. Reproducibility of plasma hormone levels in postmenopausal women over a 2-3-year period. Cancer Epidemiol Biomarkers Prev. 1995;4:649-54.

23. Missmer SA, Spiegelman D, Bertone-Johnson ER, Barbieri RL, Pollak MN, Hankinson SE. Reproducibility of Plasma Steroid Hormones, Prolactin, and Insulin-like Growth Factor Levels among Premenopausal Women over a 2- to 3-Year Period. Cancer Epidemiol Biomarkers Prev. 2006;15:972-8.

24. Koenig KL, Toniolo P, Bruning PF, Bonfrer JM, Shore RE, Pasternack BS. Reliability of serum prolactin measurements in women. Cancer Epidemiol Biomarkers Prev. 1993;2:411-4.

\section{Submit your next manuscript to BioMed Central and take full advantage of:}

- Convenient online submission

- Thorough peer review

- No space constraints or color figure charges

- Immediate publication on acceptance

- Inclusion in PubMed, CAS, Scopus and Google Scholar

- Research which is freely available for redistribution 